\title{
Infrared Spectroscopy in Molecular Study of the Piezoelectric Effect in Pig's Shin Bone
}

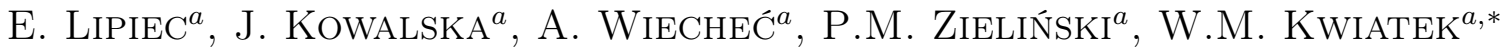
AND M. IWANIEC ${ }^{b}$

${ }^{a}$ The Henryk Niewodniczański Institute of Nuclear Physics, Polish Academy of Sciences, Kraków, Poland ${ }^{b}$ Department of Process Control, AGH University of Science and Technology, Kraków, Poland

Despite growing number of literature demonstrating the piezoelectric effect in bones at macro level there's still a lack of papers describing this effect at molecular level. In order to examine this effect more specifically, a study has been conducted to show the possibility of using infrared spectroscopy on samples contained in the electric field. This is the first known research on applying infrared spectroscopy to study the influence of electric field and compressive stress on bones' structure represented by the molecular IR spectrum. The samples used in this experiment as a model were prepared in pellet form, made from powdered pig's shin bone mixed with $\mathrm{KBr}$ (mass ratio of about 1:100). The spectra were obtained in transmission mode. Three different types of experiments were performed at each sample, which were subjected to: (a) fixed electric field $(E<40 \mathrm{kV} / \mathrm{m})$, (b) variable electric field $(0-40 \mathrm{kV} / \mathrm{m}),(\mathrm{c})$ compressive stress $(P<100 \mathrm{MPa})$. The obtained data have shown that the electric field and compressive stress on sample modified infrared spectra of the bone. Qualitative as well as quantitative changes in the spectral range between $900 \mathrm{~cm}^{-1}$ and $1200 \mathrm{~cm}^{-1}$ and the band at $562 \mathrm{~cm}^{-1}\left(\mathrm{PO}_{4}^{3-}\right.$ asymmetric and symmetric stretching, respectively) were observed. The relative area obtained by decomposition of the $\mathrm{PO}_{4}^{3-}$ band reaches an extreme value in the external compressive stress $(10 \mathrm{MPa})$, that occur under physiological conditions. Based on presented data, it was proved that the applied factors (electric field, compressive stress) can change the oscillation energy and the number of molecule's degrees of freedom.
\end{abstract}

PACS: 78.30.Jw, 07.57.Ty, 77.65.-j, 87.50.ct

\section{Introduction}

The growth and structuring of the bones are based on piezoelectric effect. An application of compressive stress to the bone surface causes the formation of two differently charged surfaces. Compressed surface becomes electropositive and streched one - electronegative [1]. Induced currents control the bone growth and the formation of trabecular structure, due to the deposition of calcium. The piezoelectric effect determines the construction of anatomical bone structures. The piezoelectric effect in bones is also used in the treatment of osteoporosis, where the noninvasive electromagnetic stimulation prevents bone loss by inducing currents, that stimulate bone formation [2-4].

However at the molecular level the piezoelectric effect is still poorly investigated. Therefore this effect was more specifically examined. This study was performed to verify that the infrared spectroscopy can be used as a tool that may help understanding the piezoelectric effect on molecular level on bone structure.

\section{Materials and method}

In this study a commercially avaliable pig's shin bone was used as a model. Prior to a bone fragmentation it

* corresponding author; e-mail: wojciech.kwiatek@ifj.edu.pl was mechanically cleaned from organic matter and not chemical treatment was applied. The samples were prepared in form of pellet, made of powdered bone mixed with $\mathrm{KBr}$ (mass ratio of about 1:100). $\mathrm{KBr}$ and the sample were mixed and powdered in agate mortar and then pressed at the $16 \mathrm{MPa}$ pressure into square pellets. The degree of fragmentation of the sample was determined by the quality of the spectra. The square pellets were prepared to gain a uniform stress distribution in the whole sample, during compression. The size of pellets (about $15 \mathrm{~mm} \times 15 \mathrm{~mm} \times 1.2 \mathrm{~mm}$ ) was optimized to get a high quality infrared spectra and not to destroy the pellets during compression under IR microscope.

\section{Measuring equipment}

The samples were studied by the Fourier transform infrared microspectroscopy (FTIR) at the globar source (Bruker IFS 66v/S, IR scope II).

During the IR measurements, each sample was measured under IR microscopy while separately subjected to:
a) fixed electric field $(E<40 \mathrm{kV} / \mathrm{m})$,
b) variable electric field $(0-40 \mathrm{kV} / \mathrm{m})$,
c) compressive stress $(P<100 \mathrm{MPa})$. 
The values of electric field and compressive stress were chosen based on literature. These parameters were used as model of natural physiological conditions [5, 6]. In living organism the inverse piezoelectric effect appears under the influence of a compressive stress value applied in the presented study. The piezoelectric effect and the inverse piezoelectric effect were not directly measured during the experiment, but the values of applied parameters (electric field and compressive stress) determine the appearance of described effects in samples during infrared measurements.

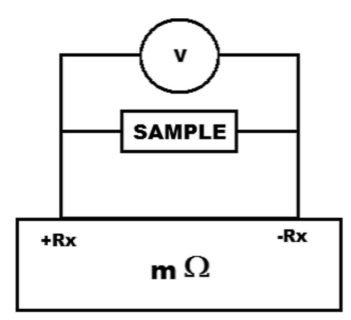

Fig. 1. Scheme of a system for applying an electric field during the measurement.

The diagram of a system for applying an electric field during measurement is presented in Fig. 1. During the measurements the sample was placed between two aluminum electrodes. The range of electric field values reached from $\approx 6 \mathrm{kV} / \mathrm{m}$ to $\approx 40 \mathrm{kV} / \mathrm{m}$.

The samples were compressed using a vise. Compression force was measured by extensometer Lorenz K25. The voltage, proportional to the applied force, was read using voltmeter. The surface of the sample, which was attached to the force, was equal to $1.8 \times 10^{5} \mathrm{~m}^{2}$. The range of compressive stress values were from $\approx 11 \mathrm{GPa}$ to $\approx 90 \mathrm{GPa}$.

\section{Analysis under IR microscopy}

The FTIR spectra of bone/KBr pellets were obtained in transmission mode, with a required resolution of $4 \mathrm{~cm}^{-1}$, in the spectral range of $400-4000 \mathrm{~cm}^{-1}$. The number of scans was set to 64 . Three different samples were investigated and in order to get a good statistics each sample was measured in 3 different places. The spectrum of pure $\mathrm{KBr}$ pellet was used as a background spectrum measure at the same conditions as the investigated sample.

\section{Spectral analysis}

Initially the second-degree polynomial baseline correction was applied and then the identification of characteristic bands was done. The analyzed bands in the infrared spectrum are shown in Fig. 2, while Table I describes their origin.

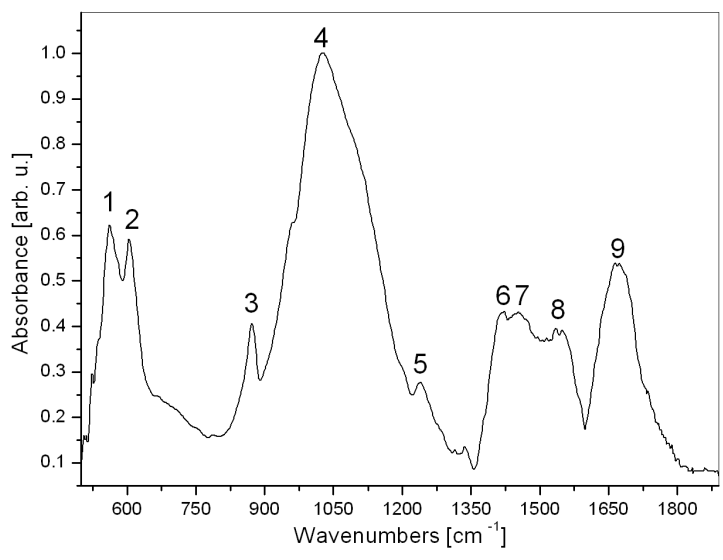

Fig. 2. Averaged (3 samples and 3 different places on each sample) spectra of control bone. Numbered bands are described in Table I.

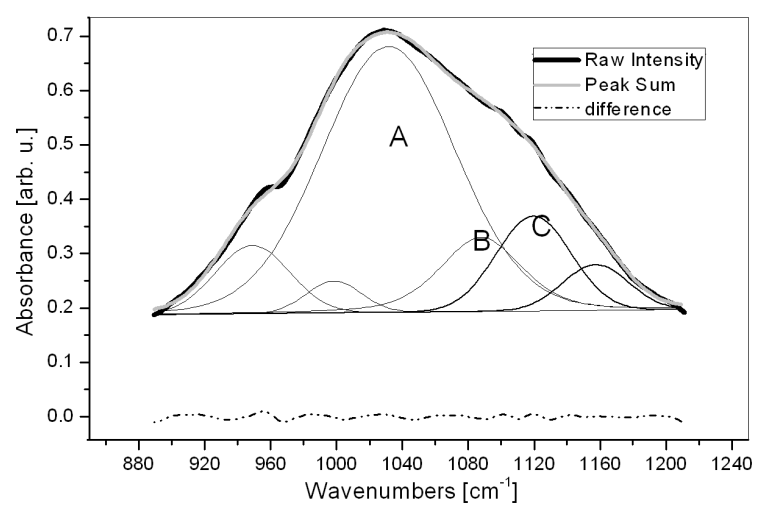

Fig. 3. Fitting analysis in the wave number region $900-1200 \mathrm{~cm}^{-1}$.

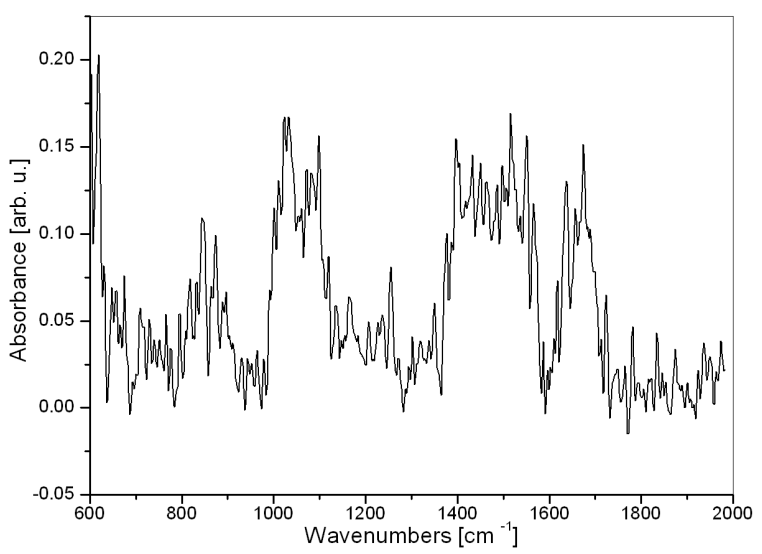

Fig. 4. IR spectrum of bone under compressive stress $\approx 90 \mathrm{GPa}$. 
Infrared band of bone in spectral range $500-1800 \mathrm{~cm}^{-1}[6-9]$.

TABLE I

\begin{tabular}{l|l|l}
\hline \hline & Wave numbers $\left[\mathrm{cm}^{-1}\right]$ & \multicolumn{1}{c}{ Assignment $[6-9]$} \\
\hline 1 & 562 & $\begin{array}{l}\mathrm{P}-\mathrm{O} \text { symmetric stretching in } \mathrm{PO}_{4}^{3-} \text { group } \\
\text { (mineral part of bone) }\end{array}$ \\
\hline 2 & 604 & $\begin{array}{l}\mathrm{P}-\mathrm{O} \text { symmetric stretching in } \mathrm{PO}_{4}^{3-} \text { group } \\
\text { (mineral part of bone) }\end{array}$ \\
\hline 3 & 872 & $\begin{array}{l}\mathrm{C}-\mathrm{O} \text { symmetric stretching in } \mathrm{CO}_{3}^{2-} \text { group } \\
\text { (mineral part of bone) }\end{array}$ \\
\hline 4 & $900-1200$ & $\begin{array}{l}\mathrm{P}-\mathrm{O} \text { symmetric and antisymmetric stretching } \\
\text { in } \mathrm{PO}_{4}^{3-} \text { group (mineral part of bones) }\end{array}$ \\
\hline 5 & 1239 & $\begin{array}{l}\mathrm{P}-\mathrm{O} \text { antisymmetric stretching in } \mathrm{PO}_{4}^{3-} \text { group } \\
\text { (mineral part of bone) }\end{array}$ \\
\hline 6 & 1422 & $\begin{array}{l}\mathrm{C}-\mathrm{O} \text { symmetric stretching in } \mathrm{CO}_{3}^{2-} \text { group } \\
\text { (amide II) }\end{array}$ \\
\hline 7 & 1453 & $\begin{array}{l}\mathrm{C}-\mathrm{N} \text { symmetric stretching, } \mathrm{H}-\mathrm{N}-\text { symmetric } \\
\text { stretching (amide II) }\end{array}$ \\
\hline 8 & 1535 & $\mathrm{C}-\mathrm{N}$ symmetric stretching (amide II) \\
\hline 9 & 1664 & $\mathrm{C}=\mathrm{O}$ symmetric stretching (amide I)
\end{tabular}

Infrared band of bone in spectral range $900-1200 \mathrm{~cm}^{-1}[9]$.

TABLE II

\begin{tabular}{|c|c|c|}
\hline & Wave numbers $\left[\mathrm{cm}^{-1}\right]$ & Assignment [9] \\
\hline $\mathrm{A}$ & $1031 \pm 15$ & $\begin{array}{l}\mathrm{P}-\mathrm{O} \text { symmetric stretching in mature, } \\
\mathrm{PO}_{4}^{3-} \text { group in } 125-190 \AA \text { hydroxyapatite crystals }\end{array}$ \\
\hline $\mathrm{B}$ & $1079 \pm 83$ & $\begin{array}{l}\mathrm{P}-\mathrm{O} \text { symmetric stretching in growing } \\
\mathrm{PO}_{4}^{3-} \text { group in } 135-150 \AA \text { hydroxyapatite crystals }\end{array}$ \\
\hline $\mathrm{C}$ & $1120 \pm 60$ & $\begin{array}{l}\mathrm{P}-\mathrm{O} \text { antisymmetric stretching in mature } \\
\mathrm{PO}_{4}^{3-} \text { group in } 125-190 \AA \text { hydroxyapatite crystals }\end{array}$ \\
\hline
\end{tabular}

The spectra were analyzed by fitting the bands with Gaussian-Lorentzian curves. Since the applied factors mostly influence on the structure of hydroxyapatite (HAp) and other calcium compounds, the band fitting was performed in the spectral range of $900-1200 \mathrm{~cm}^{-1}$ which corresponds to the $\mathrm{P}-\mathrm{O}$ symmetric and asymmetric stretching of $\mathrm{PO}_{4}^{3-}$ group of this mineral. The result of this approach is shown in Fig. 3 and the fitted bands are shown respectively in Table II. The analysis of bones under compressive stress $\approx 90 \mathrm{GPa}$ was impossible because of poor quality spectra (Fig. 4).

\section{Results}

Some changes were observed in the analyzed spectral range $\left(900-1200 \mathrm{~cm}^{-1}\right)$. This region reflects the maturation of HAp crystals [9]. The percentage of three major components in this spectra range is presented in Fig. 5 and Fig. 6. The obtained data have shown that the electric field and compressive stress have an influence on IR bone spectra. The decrease of absorbance value could be correlated with the reducing of the degrees of molecules freedom. The percentage of all components can be determined as the reactivity of functional groups in HAp crystals. The influence of electric field or compressive stress on $\mathrm{O}-\mathrm{P}$ symmetric and antisymmetric stretching depends on the size of hydroxyapatite crystals. Smaller crystals are more sensitive to electric field and compressive stress. An influence of applied factors on mature hydroxyapatite crystals was not observed. Probably it can be correlated with the mineralization and growth of the bones. Component $\mathrm{B}$ originates from small hydroxyapatite crystals (135-150 $\AA$ ). The percentage of component $\mathrm{B}$ has the minimum value of used factors as obtained in physiological conditions.

\section{Conclusions}

Based on described results, it was proved that the applied parameter such as electric field and compressive 


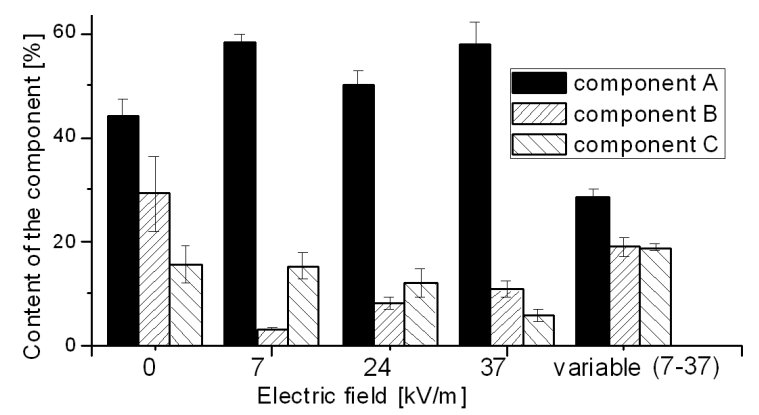

Fig. 5. Percentage of the component in the spectral range from $900 \mathrm{~cm}^{-1}$ to $1200 \mathrm{~cm}^{-1}$, the samples were treated by fixed and variable electric field.

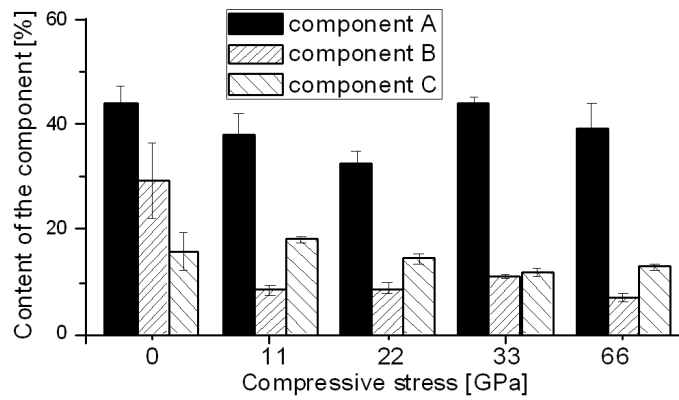

Fig. 6. Percentage of the component in the spectral range from $900 \mathrm{~cm}^{-1}$ to $1200 \mathrm{~cm}^{-1}$, the samples were treated by compressive stress. stress can change the oscillation energy and the number of molecules freedom degrees. The infrared spectroscopy is useful tool to investigate the piezoelectric effect on molecular level.

\section{References}

[1] E. Fakuda, I. Yasuda, J. Phys. Soc. Jpn. 12, 1158 (1957).

[2] G. Aschero, F. Gizdulich, S. Mango, M. Romano, J. Biomech. 29, 1169 (1996).

[3] K. Noris-Suárez, J. Lira-Olivares, A.M. Ferreira, A. Graterol, J.L. Feijoo, S.W. Lee, Mater. Sci. Forum 981, 544 (2007).

[4] A.L. Boskey, Curr. Osteoporos. Rep. 4, 71 (2006).

[5] K. Li, Y. Hui, S. Ma, G. Ding, Y. Guo, J. Liu, Y. Li, G. Guo, Prog. Electromagn. Res. 112, 139 (2011).

[6] N. Ozkaya, M. Nordin, Fundamentals of Biomechanics: Equilibrium, Motion and Deformation, Springer-Verlag, New York 1999.

[7] H. Boyar, F. Zorlu, M. Mut, F. Severcan, Anal. Bioanal. Chem. 379, 433 (2004).

[8] C. Chadefaux, A. Le Hô, L. Bellot-Gurlet, I. Reiche, e-Ps 6, 129 (2009).

[9] N. Pleshko, A. Boskey, R. Mendelsohn, Biophys. J. 60, 786 (1991). 